decreases from $2.5 \%$ of total body weight in young people to about $1.6 \%$ in the nonagenarian population, and several morphological changes with aging indicate that the liver cells in advanced old age are in a hyperfunctioning state, possibly trying to compensate for the decline in total cell number. ${ }^{4}$ However, it has been suggested that aging has a limited effect on liver functions but more on its response to extrahepatic factors, ${ }^{5}$ disease states or increased metabolic demands to which elderly people may have an impaired ability to respond. ${ }^{4}$

Atheromatosis usually affects the celiac trunk in old donors but, although this happens rarely, when atheroma occurs distally at the level of the right hepatic artery or at the bifurcation of the gastroduodenal and the common hepatic artery, the liver graft must be discarded for LT. ${ }^{2}$

The LT procedure is a scenario in which many donor risk factors may be involved. Thus, in our previous brief communication on octogenarian liver donors, we recommended the use of liver grafts with no age limit but in good preprocurement condition (hemodynamic stability, low doses of vasopressor drugs), short intensive care unit stay, good liver function tests, soft liver consistency, absence of hepatic artery atheromatosis, cold ischemia time less than 9 hours, and macrosteatosis less than $30 \% .{ }^{1}$ From that time, we have continued applying the same criteria, but in recent years we have added other conditions such as implanting the octogenarian livers into recipients without Hepatitis $C$ virus (HCV) cirrhosis and model for end-stage liver disease scores lower than 20 who could tolerate well a probable transient graft dysfunction. ${ }^{2}$ As we have seen in this short experience, it is possible that an octogenarian liver graft can reach the centennial age after transplant while the recipient maintains a normal liver function and a good quality of life if a good donor selection is done, and recipients with HCV cirrhosis or advanced liver disease are excluded, but at present, with the current introduction of new anti-HCV drugs, we are also using these older donors in HCV recipients to decrease the waiting list mortality. However, further investigations are needed to demonstrate good results using octogenarian liver donors in HCV recipients.

\section{REFERENCES}

1. Jiménez Romero C, Moreno González E, Colina Ruíz F, et al. Use of octogenarian livers safely expands the donor pool. Transplantation. 1999;68: 572-575.

2. Jiménez-Romero C, Caso Maestro O, Cambra Molero F, et al. Using old liver grafts for liver transplantation: where are the limits? World J Gastroenterol. 2014;20:10691-10702.

3. Mooney H, Roberts R, Cooksley WG, et al. Alterations in the liver with ageing. Clin Gastroenterol. 1985;14:757-771.

4. James OF. Gastrointestinal and liver function of old age. Clin Gastroenterol. 1983;12:671-691.

5. Popper H. Coming of age. Hepatology. 1985;5:1224-1226.

\title{
Torque Teno Virus Load and Acute Rejection After Orthotopic Liver Transplantation
}

Federico Simonetta, MD, PhD, ${ }^{1}$ Amandine Pradier, $\mathrm{PhD},{ }^{1}$ Stavroula Masouridi-Levrat, MD, ${ }^{1}$ Christian van Delden, MD, ${ }^{2}$ Emiliano Giostra, MD, ${ }^{3}$ Isabelle Morard, MD, ${ }^{3}$ Nicolas Mueller, MD, ${ }^{4}$ Beat Muellhaupt, MD, ${ }^{5}$ Piero V. Valli, MD, ${ }^{5}$ Nasser Semmo, MD, ${ }^{6}$ Jörg Seebach, MD, ${ }^{7}$ Yves Chalandon, MD, ${ }^{1}$ Laurent Kaiser, MD, ${ }^{2}$ Eddy Roosnek, PhD, ${ }^{1}$ and Swiss Transplant Cohort Study (STCS)

eplication of torque teno virus (TTV), a nonpathogenic, highly prevalent anellovirus, increases considerably during immunesuppression. ${ }^{1,2}$ Recently, Schiemann and

Received 30 November 2016. Revision received 24 January 2017.

Accepted 12 February 2017.

${ }^{1}$ Division of Hematology, Department of Medical Specialties, Geneva University Hospitals, Geneva, Switzerland.

${ }^{2}$ Division of Infectious Diseases, Department of Medical Specialties, Geneva University Hospitals, Geneva, Switzerland.

${ }^{3}$ Division of Gastroenterology and Hepatology, Department of Medical Specialties, Geneva University Hospitals, Geneva, Switzerland.

${ }^{4}$ Division of Infectious Diseases and Hospital Epidemiology, University Hospital Zurich, Zurich, Switzerland.

${ }^{5}$ Department of Gastroenterology and Hepatology, University Hospital Zürich, Zürich, Switzerland.

${ }^{6}$ Hepatology Unit, Department of Visceral Surgery and Medicine University Hospital Bern, Bern, Switzerland.

${ }^{7}$ Division of Immunology and Allergy, Department of Medical Specialties, Geneva University Hospitals, University of Geneva, Geneva, Switzerland. coworkers published the inverse association of TTV load with rejection after kidney transplantation. ${ }^{3}$ We report similar findings in 39 patients (median age, 60 years [range, 1-73 years];

The authors declare no funding or conflicts of interest.

F.S.: designed the study, analyzed and interpreted the data, and wrote the manuscript. A.P.: performedl experiments, analyzed data, and edited and approved the manuscript. S.M.L., C.V.D., E.G., I.M., N.M., B.M., P.V., N.S., J.S., and Y.C.: provided substantial contributions to the conception of the work, participated in clinical data collection, and edited and approved the manuscript. L.K.: provided substantial contributions to the conception of the work and to acquisition of data, interpreted the data, helped to write and approved the manuscript. E.R.: interpreted the data, provided overall research supervision, and wrote the manuscript.

Correspondence: Federico Simonetta MD, PhD, Division of Hematology, Department of Medical Specialties, Geneva University Hospitals, Rue GabriellePerret-Gentil 4, 1205 Geneva, Switzerland. (federico.simonetta@hcuge.ch).

Copyright (C) 2017 Wolters Kluwer Health, Inc. All rights reserved.

ISSN: 0041-1337/17/10107-e219

DOI: 10.1097/TP.0000000000001723 
male subjects, 59\%) enrolled in the Swiss Transplant Cohort Study after orthotopic liver transplantation (OLT) for liver disease of viral $(33 \%)$, toxic $(10 \%)$, or other $(57 \%)$ origins.

We measured TTV-DNA levels by real-time polymerase chain reaction ${ }^{4}$ at time of transplantation and at 6 and 12 months posttransplant for the 19 patients with available serum samples. Seventy-four healthy subjects served as controls (HC). TTV-DNA was detectable (detection limit 25 viral copies $/ \mathrm{mL}$ of plasma) in $29(74 \%)$ of 39 patients at transplantation, a prevalence similar to the one observed in controls $\left(51 / 74,69 \% ; P=0.8035 ; \chi^{2}\right.$ test). However, median TTV-DNA levels were significantly higher in OLT recipients (median, $4.54 \times 10^{3}$ copies $/ \mathrm{mL}$; range, $25-1.2 \times 10^{8}$ ) compared with controls (median, $1.7 \times 10^{2}$ copies $/ \mathrm{mL}$; range, 25-5.4 $\times 10^{4} ; P=0.0014$, Mann-Whitney $U$ test) (Figure 1A). As shown previously, ${ }^{1,2}$ TTV titers in OLT recipients increased significantly after transplantation (median, $2 \times 10^{6}$; range, $25-5.4 \times 10^{8}$ at 6 months; and median, $2.2 \times 10^{5}$; range, $25-1.7 \times 10^{7}$ at 12 months) (Figure $1 \mathrm{~A}$ ).

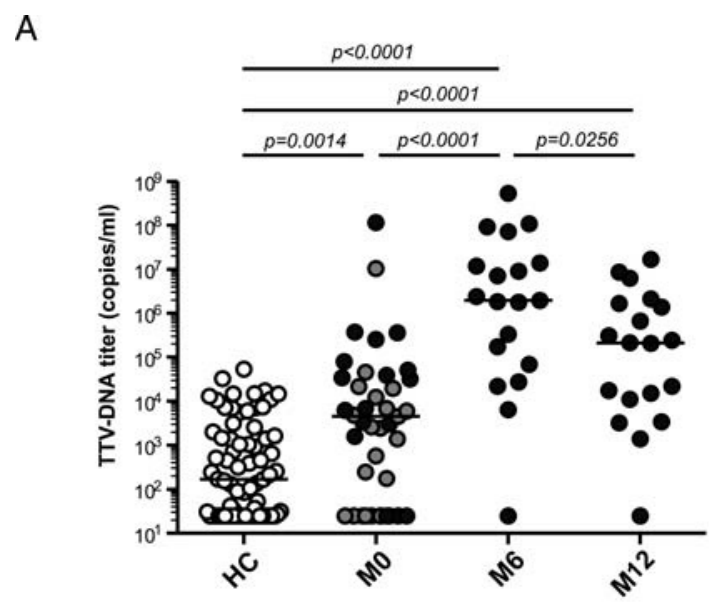

B

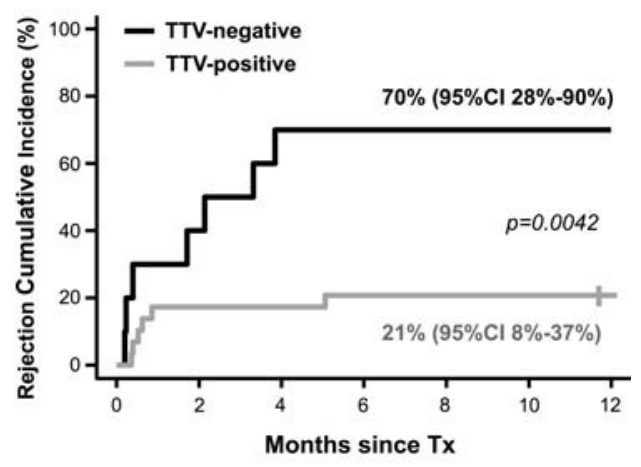

\begin{tabular}{lccccccc}
\multicolumn{7}{c}{ Number at risk } \\
TTV-negative & 10 & 6 & 3 & 3 & 3 & 3 & 3 \\
TTV-positive & 29 & 23 & 23 & 22 & 21 & 21 & 20
\end{tabular}

FIGURE 1. TTV load kinetics and impact on the frequency of rejection episodes after OLT. A, TTV-DNA titers in OLT recipients (filled dots) compared with healthy controls ( $\mathrm{HC}$, white dots). Gray filled dots identify patients analyzed at time of transplantation (MO) exclusively. Black filled dots identify patients analyzed at time of transplantation (MO) and 6 (M6) and 12 months (M12) after transplantation. Comparison was performed using the Mann-Whitney $U$ test, and $P$ values are indicated when significant $(P<0.05)$. B, One-year biopsy-proven acute rejection cumulative incidence in patients displaying detectable (gray line) and undetectable (black line) TTV-DNA at transplantation. Cumulative incidence of rejection analysis was performed using Gray test with death without rejection as a competing event.
We further assessed the relationship between TTV-loads at transplantation with the risk of biopsy-proven acute cellular graft rejection (Rejection Activity Index score $\geq 3$ or $\geq 2$ with significant necrosis) during the first year after transplantation. Interestingly, 1-year cumulative incidence of rejection in OLT recipients with detectable TTV-DNA plasma levels at transplantation was significantly lower (21\% [95\% confidence interval (CI), 8\%-37\%]) than in patients with undetectable TTV titers $(70 \%$ [95\% CI, $28 \%-90 \%] ; P=0.0042$, Gray test; Figure 1B).

To account for factors with potential impact on TTV replication and rejection such as age, gender, $\mathrm{HBV}, \mathrm{HCV}$, and HIV serostatus, underlying disease, number of immunosuppressive drugs used, hepatic encephalopathy, and presence of human leukocyte antigen donor-specific antibodies, we performed a multivariate analysis using FineGray proportional hazard regression for competing events. This analysis confirmed the reduced 1-year cumulative incidence of rejection in patients with detectable TTV titers at time of transplantation (HR, $9 \times 10^{-3}\left[95 \% \mathrm{CI}, 1 \times 10^{-3}\right.$ to $\left.9.2 \times 10^{-2} ; P=0.00008\right]$ ).

Our results in OLT recipients are in agreement with those reported by Schiemann et al for kidney transplant recipients and reinforce the results of De Vlaminck and coworkers who found that anellovirus levels in heart and lung transplant recipients with acute rejection episodes were lower than in nonrejecting patients. ${ }^{5}$ It is tempting to speculate that a higher immunocompetence in TTV-negative patients at transplantation could be responsible for the higher incidence of rejection episodes observed during the first year after transplantation.

Obviously, our study presents several limitations. First, because serological testing for TTV is currently unavailable, we cannot exclude that some patients are TTV-negative simply because they never have been infected. However, we do believe that the high prevalence of TTV in the population makes this unlikely. Second, the study remains somewhat incomplete because the lack of posttransplant serum samples precluded the analysis of a potential prognostic value of TTV titers after OLT. Larger prospective studies with more patients than in our retrospective pilot study are needed to assess whether monitoring TTV titers could help identifying patients at higher risk of acute rejection after OLT.

\section{ACKNOWLEDGMENTS}

This study has been conducted in the framework of the Swiss Transplant Cohort Study, supported by the Swiss National Science Foundation and the Swiss University Hospitals (G15) and transplant centers.

Swiss Transplant Cohort Study (STCS) Members: Rita Achermann, Patrizia Amico, John-David Aubert, Vanessa Banz, Guido Beldi, Christian Benden, Christoph Berger, Isabelle Binet, Pierre-Yves Bochud, Heiner Bucher, Thierry Carell, Emmanuelle Catana, Yves Chalandon, Sabina de Geest, Olivier de Rougemont, Michael Dickenmann, Michel Duchosal, Laure Elkrief, Thomas Fehr, Sylvie Ferrari-Lacraz, Christian Garzoni, Paola Gasche Soccal, Christophe Gaudet, Emiliano Giostra, Déla Golshayan, Karine Hadaya, Jörg Halter, Dominik Heim, Christoph Hess, Sven Hillinger, Hans H. Hirsch, Günther Hofbauer, Uyen Huynh-Do, Franz Immer, Richard Klaghofer, Michael Koller (Head of the data center), Bettina Laesser, Roger Lehmann, Christian Lovis, 
Pietro Majno; Oriol Manuel, Hans-Peter Marti, Pierre Yves Martin, Pascal Meylan (Head, Biological Samples Management Group), Paul Mohacsi, Philippe Morel, Ulrike Mueller, Nicolas J Mueller (Chairman, Scientific Committee), Helen Mueller-McKenna (Head, Local Data Management), Antonia Müller, Thomas Müller, Beat Müllhaupt, David Nadal, Manuel Pascual (Executive Office), Jakob Passweg, Juliane Rick, Eddy Roosnek, Anne Rosselet, Silvia Rothlin, Frank Ruschitzka, Urs Schanz, Stefan Schaub, Aurelia Schnyder, Christian Seiler, Susanne Stampf, Jürg Steiger (Head, Executive Office), Guido Stirnimann, Christian Toso, Christian Van Delden (Executive Office), Jean-Pierre Venetz, Jean Villard, Madeleine Wick (STCS Coordinator), Markus Wilhelm, and Patrick Yerly.

\section{REFERENCES}

1. Shang D, Lin Y, Rigopoulou I, et al. Detection of $\Pi$ virus DNA in patients with liver disease and recipients of liver transplant. J Med Virol. 2000;61: 455-461.

2. Béland K, Dore-Nguyen M, Gagné M-J, et al. Torque teno virus in children who underwent orthotopic liver transplantation: new insights about a common pathogen. J Infect Dis. 2014;209:247-254.

3. Schiemann M, Puchhammer-Stöckl E, Eskandary F, et al. Torque teno virus load-inverse association with antibody-mediated rejection after kidney transplantation. Transplantation. 2017;101:360-367.

4. Masouridi-Levrat S, Pradier A, Simonetta F, et al. Torque teno virus in patients undergoing allogeneic hematopoietic stem cell transplantation for hematological malignancies. Bone Marrow Transplant. 2016;51: 440-442.

5. De Vlaminck I, Khush KK, Strehl C, et al. Temporal response of the human virome to immunosuppression and antiviral therapy. Cell. 2013;155: 1178-1187.

\title{
Successful Transplantation of 2 Discolored Kidneys Caused by Myoglobin Casts From a Donor With Rhabdomyolysis
}

\author{
Chuan-bao Chen, MD, ${ }^{1}$ Jian Zhou, MD, PhD, ${ }^{1}$ Xiao-ping Wang, MD, PhD, ${ }^{1}$ Ming Han, MD, PhD, ${ }^{1}$ \\ Wen-fang Chen, MD, $\mathrm{PhD},{ }^{2}$ and Xiao-peng Yuan, $\mathrm{MD}, \mathrm{PhD}^{1}$
}

A discolored/black kidney has 5 major differential diagnoses: massive microthrombi and/or severe cortical necrosis, hemosiderosis, lipofuscin or melanin pigment deposits, and oculocutaneous albinism. Renal hemosiderosis is an indicator of severe intravascular hemolysis. ${ }^{1}$ Lipofuscin or melanin pigment deposits can also cause black kidney. ${ }^{2,3}$ In addition, oculocutaneous albinism can result in black kidneys due to accumulation of ceroid-like material in the lysosomes of the kidney. ${ }^{4}$ Herein, we report 2 black kidneys which resulted from a donation after cardiac death (DCD) donor with severe rhabdomyolysis which were successfully transplanted.

An 18-year-old man was severely injured in a motor vehicle accident. His medical history was unremarkable, without

Received 20 December 2016. Revision received 7 February 2017.

Accepted 20 February 2017.

${ }^{1}$ Department of Organ Transplantation, First Affiliated Hospital of Sun Yat-sen University, Guangzhou, P. R. China.

${ }^{2}$ Department of Pathology, First Affiliated Hospital of Sun Yat-sen University, Guangzhou, P. R. China.

The authors declare no funding or conflicts of interest.

C.C. and X.Y. collected data and wrote the article. M.H., X.W., and J.Z. collected data and participated in critical review of the article, W.C. performed pathology review.

Correspondence: Xiao-peng Yuan, MD, PhD, Huangpudong Road 183, Guangzhou 510700, P.R. China. (transplantyuan@163.com).

Copyright (C) 2017 Wolters Kluwer Health, Inc. All rights reserved.

ISSN: 0041-1337/17/10107-e221

DOI: 10.1097/TP.0000000000001717 chronic anemia, oculocutaneous albinism or other related syndromes. He was hospitalized in the intensive care unit for 26 days before donation. His initial laboratory results were as follows: serum creatinine (SCr), $0.6 \mathrm{mg} / \mathrm{dL}$; creatine phosphokinase (CPK), 433 units/L; and serum myoglobin, $540 \mu \mathrm{g} / \mathrm{L}$. His peak CPK was 22408 units/L, peak serum myoglobin was $119617 \mu \mathrm{g} / \mathrm{L}$, and peak SCr $4.4 \mathrm{mg} / \mathrm{dL}$. At the time of organ procurement, his $\mathrm{SCr}$ was $1.3 \mathrm{mg} / \mathrm{dL}$, CPK was 439 units/L, and serum myoglobin was $353 \mu \mathrm{g} / \mathrm{L}$. Urine output for the 24 hours before organ procurement was $3550 \mathrm{~mL}$, and the terminal urine output was $150 \mathrm{~mL} / \mathrm{h}$. Organs were donated through the procedure of DCD. The functional warm ischemic time (starts when the systolic blood pressure has a sustained fall below $50 \mathrm{~mm} \mathrm{Hg}$ and extends up to the onset of cold in situ perfusion) was 6 minutes. The time from oxygen saturation falls below $70 \%$ to the onset of cold in situ perfusion was 15 minutes. After finishing cold in situ perfusion, the kidneys looked black (Figure 1A). Based on the black appearance, other centers turned down the kidneys. Wedge biopsy was performed on the right kidney, and frozen section showed normally structured glomeruli and tubules, mild edema of tubular epithelial cells, and no microthrombosis in the vessel loop of the glomeruli. The right kidney was preserved by static cold storage, and the left kidney was preserved by a LifePort Organ Recovery Systems, the pump perfusion lasted for 16 hours with terminal flow $141 \mathrm{~mL} / \mathrm{min}$ and resistance $0.16 \mathrm{~mm}$ of $\mathrm{Hg} /(\mathrm{mL} / \mathrm{min})$. Based on the donor's history, the renal biopsy pathological results, and the pump characteristics, the 2 kidneys were accepted for transplantation at our center. 